\title{
Failure Analysis of Au-AI Wire Bonding to MOSFETs
}

\section{JiaoyingHuang ${ }^{\mathrm{a}}, \mathrm{CanCui}^{\mathrm{b}}, \mathrm{ChengGao}^{\mathrm{c}}$ andYuanyuanXiong ${ }^{\mathrm{d}}$}

School of Reliability and System Engineering, BeihangUniversity, Beijing 100191, China.

ahuangjy@buaa.edu.cn, ${ }^{b}$ cuican1414@buaa.edu.cn, ${ }^{\mathrm{C}}$ gaocheng@buaa.edu.cn, ${ }^{\mathrm{d}}$ zhenghao825@126 .com

Keywords: Failure analysis, MOSFET,Au-Al wire bonding,reliability, bonding strength.

\begin{abstract}
The gold and aluminum (Au-Al) wire bonding is used to realize interconnection for semiconductor devices. But, sometimes, the failure of $\mathrm{Au}-\mathrm{Al}$ bond wire exists. In this paper, the failure influence factors of Au-Al bonding to Metal-Oxide-Semiconductor Field-Effect Transistors (MOSFETs) were discussed. Then the wire bond failure analysis of MOSFETs were researched. And bond strength analysis, microscopic examination, scanning electron microscopy (SEM) examination and energy dispersive spectrometer (EDS) analysis, which belongs to the details of all the tests, were completed combined with an application example of MOSFETs. The results demonstrated that the high temperature and the stain in the device lead to the failure of MOSFETs. The temperature is too high to make voids named Kirkendall holes on the interface of Au-Al bonding. And the pollution influenced the materials to cause the bond wire off the substrate. It was suggested that the key parameter, that it to say, the temperature and the pollution like carbon oxygen compound should be strictly controlled to improve the reliability of MOSFETs, especially in the high temperature storage and working conditions.
\end{abstract}

\section{Introduction}

As the basis for system, equipment and products, components play an increasingly important role in them [1]. So, in recent years, the quality and reliability of components are concerned by makers and users. And the wire bonding technology is the most common interconnection technology of semiconductor devices. So the integrity of wire bonding technology in semiconductor devices is particularly significant [2]. The technology has two steps. First of all, both ends of the wire are welded on the chip surface and the substrate through the external force. And then, the surface of medal, which occurs the plastic deformation and molecular diffusion, are welded together by applying pressure and welding energy. And there are many different types in accordance with the multiple classification criteria. There are ball bonding and wedge bonding by the dissimilar forms of the capillary. But according to the differences of heating and ultrasonic, the wire bonding can be divided into four processes: hot pressing bonding, ultrasonic bonding, hot and ultrasonic bonding and micro gap bonding. While it also can be distinguish into copper wire bonding, wire bonding and wire bonding based on wire materials [3,4,5].

The purpose and significance of the wire bonding failure analysis may be varied in different situations. While the most direct objective is to find the cause of failure, and then to put forward prevention and preventive measures or technical plans about the same or similar devices on the basis of the cause. Finally, ensuring the reliability of components which can work normally. The basic contents of the failure analysis are that "it is necessary to know very well about the object which is analyzed, clearly determine the failure mode, carefully study the failure mechanism, judge the causes of failure and suggest some improve measures and useful recommendations" [6]. The wire bonding failures were happened because of many factors, including the parameters of bond wire, materials, temperature, stress, pollution. The inconsistent sizes of solder joint, falling second solder, the squeezed pad medal layer, the unwelded outer wire and the cracked Silicon chip are the failure modes of wire bonding. As we all know, aluminum alloy is usually used to make the Ohmic contact for semiconductor devices. And the wire frame and the electrical connections are often completed by Au-Al wire bonding. Yeoh Lai Seng,Yian-Liang Kuo, Wang Quan[7,8,9] summarized the causes of failure of $\mathrm{Au}-\mathrm{Al}$ wire bonding. The high temperature made the interface 
of the Au-Al bonding produce gold aluminum compound and come out voids and crack called Kirkendall holes.

Though the high temperature can seriously affect the function on the Au-Al wire bonding, the reason of the cracks may be more than one besides the high temperature. Au-Al wire bonding is usually used for the electrical interconnection of MOSFET. MOSFET is one which can be widely used in a variety of the analog circuit, the digital circuit, amplifier and microwave circuits, and etc. of field effect transistor (FET) [10].It can be made into enhancement or depletion type, P-channel or $\mathrm{N}$-channel with a total of 4 types. MOSFET, which is a semiconductor discrete devices, has small size, high input impedance, low noise and power consumption, high limit frequency and long life advantages.

In this paper, a kind of MOSFET, called LYNM1230T of $80 \mu \mathrm{m}$ aluminum wire-bonded silicon N-channel enhancement type, was chosen to identify their qualities of the bonding. The devices, who were packaged by TO-257 and made in China were never made tests before they were produced.Firstly, the high temperature storage test were carried out for the MOSFETs. And then bond strength test were done. From the tests above, some devices, which did not meet the standard requirements, were found. Then analyzing the causes of failure about the failure devices through observation, testing and analysis. According to the failure mode and the failure mechanism, there were some problems tojudge after the high temperature test. Generally speaking, the main cause of the failure was high temperature of the high temperature storage test.The high temperature, whichmade the surface of Au-Al wire bonding occur Kirkendall holes, lead to the MOSFETs fail.

\section{Failure Analysis about Au-Al Wire Bonding to MOSFETs}

MOSFET belongs to semiconductor discrete device. And about 25\% 30\% failure devices of semiconductor discrete device were caused by bond wire [3]. So bond wire has great effect on the reliability for the use of MOSFETs. It is extremely useful and helpful to research the failure mechanism for searching the failure reasons, preventing the failure of the device and improving their reliability. There are mainly four kinds of failure mechanism about $\mathrm{Au}-\mathrm{Al}$ wire bonding to MOSFETs

The Failure Mechanism Au-Al Wire Bonding to MOSFETs .There are 4 kinds of failure models: process error, thermal cycling, $\mathrm{Au}-\mathrm{Al}$ compound and the pollution. And the failure mechanisms are as the follows:

1) Process ErrorCaused the Failure.

a) The tail of the bond wire is too long.

b) The wire bonding off the standard position.

c) The wire bonding off the standard position makes the current density increase, and the wire bonding of E electrode is melted to form a molten crater.

d) The bond wire is too tight.

e) The lead tight and a sharp edge of ceramic tube cut off the aluminum wire.

2) Thermal Cycling Causes the Fatigue Crack of the Wire Bonding.

Silicon aluminum wire is affected by thermal cycling (or high and low temperature impact). The wire expands when heated, contracts when cooled and is repeated bending, resulting in metal fatigue and failure.

3) Au-Al Compound Caused the Failure.

There are two kinds failure mechanism about Au wire and Al substrate bonding or Al wire and pin plated Au pressure welding. On one hand, there are a series of compounds to produce because the high temperature. On the other hand, the strength of Au-Al bonding occurs low and the property of the Au-Al bond is brittle, which leads to the increase of the contact resistance and Vces because of the long term use and storage(especially above $200^{\circ} \mathrm{C}$ ).

There are some reasons to cause the Failure. The diffusion coefficient of gold is larger than aluminum, the gold is missing from the bonding surface to form many holes called Kirkendall holes and the brittle metal oxides are generated on the surface of bonding. Such as $\mathrm{Au}_{2} \mathrm{~A} 1$, 
$\mathrm{AuAl}, \mathrm{AuAl}_{2}, \mathrm{Au}_{4} \mathrm{~A} 1, \mathrm{Au}_{5} \mathrm{Al}$. This compound causes cracks which is produced the bonding region, reduced the contact area between the bond wire and medal pad and decreased the bonding strength [11].

4) The Pollution Caused the Failure.

a) The formation of aluminum film because of spit, brine, salt mist, resulting in the corrosion of the fracture and the crack of the wire.

b) Shell plating after the cleaning is not clean. The wire is corrosion and open after long term storage, even the white hair comes out around the wire.

c) Shell and pin are not clean. Gold coated pinsare in contact with the aluminum wire, and the aluminum wire is stain.

d) Gold aluminum original battery is formed owing to the leakage of package, poor quality of gold plating.So the aluminum wire is corrosion of $\mathrm{Al}(\mathrm{OH})_{3}$, long "white hair" open circuit failure.

The Failure Analysis Steps of Au-Al Wire Bonding to MOSFETs.It is very necessary for many components to do high temperature storage tests or steady-state life tests. Then after that, when the components are done for mechanical tests, there are many phenomena to come out. So it is very essential and important, according to the real circumstancesand conditions, to analyze the background ofthe devices,make wire bonding strength analysis, get on internal visual inspection, and do SEMexamination and EDSanalysis.

After high-temperature storage life tests or steady-state life tests, destructive tests are usually made to identify the bonding quality. That is to say, opening the package and make bond tension test, bond pull tests and bond shear tests included.Of course, the four main positions of disconnected wire should be confirmed in destructive tests [1]. They are as follows: the interface of the first bond, the neck of the first bond, thesecond bond and the middle of bond wire. Bond tension test is to measure the minimum separation thrust between wires and pads through horizontally pushing the wire of bonding points. However, the shear test may error due to different reasons for the test environment or human. In GJB128A-1997, there are clear acceptance criteria that bond wires of various diameters can withstand tensile values.GJB128A-1997 method 2037 Bond Strength (program A: the tension of wire, double-bonded points) shows that $88.0 \mathrm{mN}$ is the threshold of tension test for $80 \mu \mathrm{m}-\mathrm{Al}$ bond wire.

Microscopic examination, that is internal visual inspection, was to verify the structure and process of MOSFETs.

The purpose of SEM examination and EDS analysis were to analyze the components of samples and observe the defects in the process. Such as contaminants, scratches, blotchy metal, empty, nicks, cracks, dents, or tunnels.

\section{TheCase Analysis ofMOSFETs}

Eleven samples of MOSFETs were selected. They, whose type were LYNM1230T of $80 \mu \mathrm{m}$ aluminum wire-bonded silicon $\mathrm{N}$-channel enhancement, were packaged by TO-257.And all the products were never done tests after made in China.

Steady-State Life Test.All products were operated steady-state life tests. The condition of the test is under normal stress level. The temperature is $125^{\circ} \mathrm{C}$, and the period is 1000 hours.

After the test, all the samples were observed by metallurgical microscope and stereoscopic microscope, but no abnormalities were found.

Bond Tension Test.The packages of the samples were opened and a destructive bonding tension test were made. The results of the tests were shown in Table 1.

There were nine samples to meet the standard of bearing $88.0 \mathrm{mN}$. The average force was $365.1 \mathrm{mN}$. Of the remaining two samples, one had no force, and the other was $82.6 \mathrm{mN}$ smaller than the $88.0 \mathrm{mN}$. So it was considered to fail. And the position of the crack wire was the second wire bonding off the pad. After the steady-state life tests for the samples, the mechanical strengths of the bond wires were mostly good, and the partial devices had the second bond off the pads. 
The temperature, stress, time, strengths and radian of bonding have great influence on wire bonding. But the off of bonding is not only related to the bonding parameters, but also to the surface of the bonding zone and the packaging atmosphere [12]. From the data of the bonding strength test and the position of fractured wire, it is seen that some factors made the strength of the second bond low. The number of the failure samples were $8 \#$ and 11\#, then internal visual inspection, SEM examination and spectrum analysis were following.

Table 1 The results of the bond tension tests

\begin{tabular}{cccc}
\hline Numbers & Force $(\mathrm{mN})$ & Results & The positions of crack \\
\hline $1 \#$ & 441.4 & Pass & $1^{\mathrm{a}}$ \\
$2 \#$ & 453.4 & Pass & 1 \\
$3 \#$ & 308.2 & Pass & 1 \\
$4 \#$ & 407.4 & Pass & 1 \\
$5 \#$ & 361.3 & Pass & 1 \\
$6 \#$ & 357.9 & Pass & 1 \\
$7 \#$ & 353.7 & Pass & 1 \\
$8 \#$ & 82.6 & Fail & $2^{\mathrm{b}}$ \\
$9 \#$ & 318.6 & Pass & 1 \\
$10 \#$ & 283.8 & Pass & 1 \\
$11 \#$ & 0 & Fail & 2 \\
\hline
\end{tabular}

"a"indicates that the position of the crack wire is internal neck retraction point.

"b" indicates that the position of the crack wire is the pad.

Internal Visual Inspection. For the failure samples,the results of the internal appearances were shown inFig 1 and Fig 2.The Figs1 (a) and 2 (a) indicated the internal appearances about the MOSFETs. The picture shew that there were three bond wire. Two of them were thick and one was thin. There were nothing around the bonding. The first bond were good (Figs 1(b) and 2(b)).

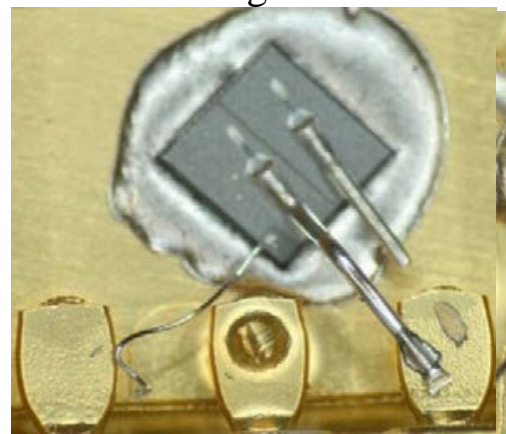

(a)

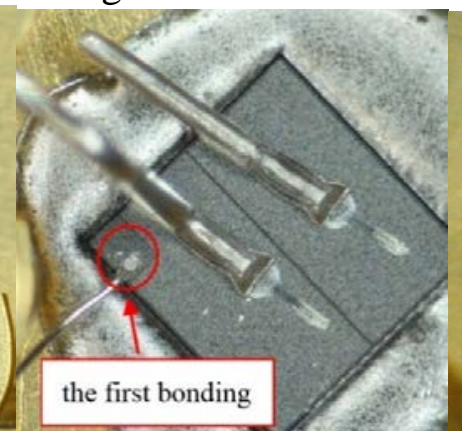

(b)

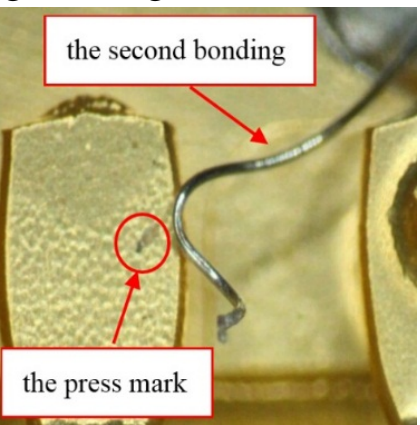

(c)

Fig 1 The internal appearances of sample11\#.(a) The internal appearances of 11\#.(b) The picture of the first bond for 11\#. (c)The picture of the second bond and the pressed mark for 11\#.

As shown in Figs 1(c) and 2(c), the second bond were wedge shape and off from the metal pads. The pressed mark of the second bond were shallow. Because the devices were well preserved, the error operation made the bond wire bend before or after the package.

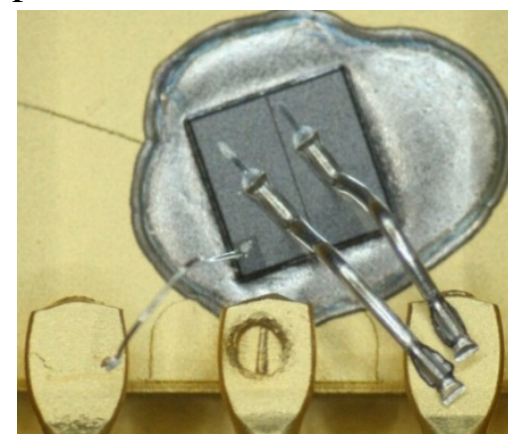

(a)

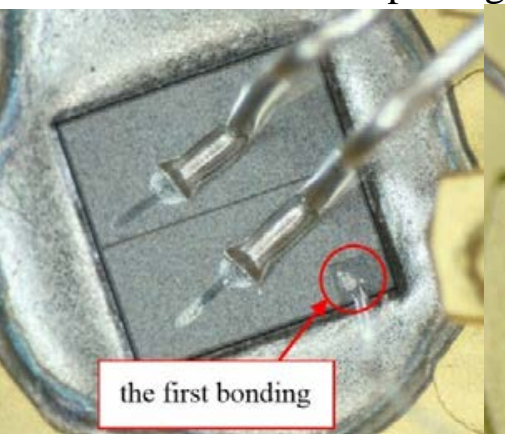

(b)

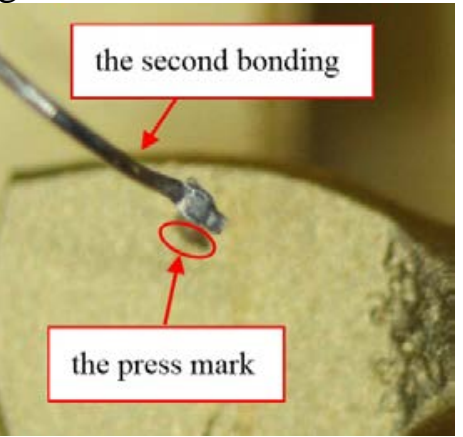

(c)

Fig 2 The internal appearances of sample 8\#.(a) The internal appearances of 8\#.(b) The picture of the first bond for 8\#. (c)The picture of the second bond and the pressed mark for8\#. 
SEM Examination. The results of SEM examination for failure samples wereexhibited inFig 3 andFig 4.

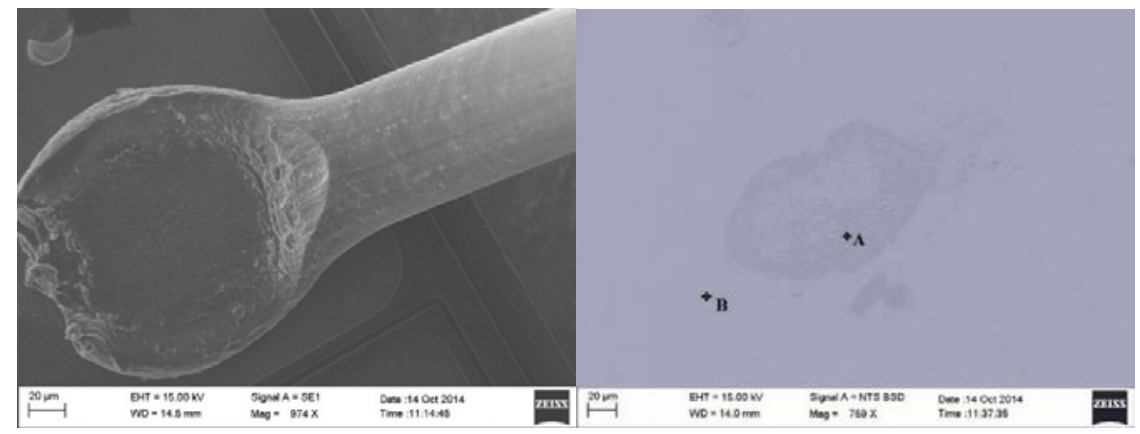

(a)

(b)

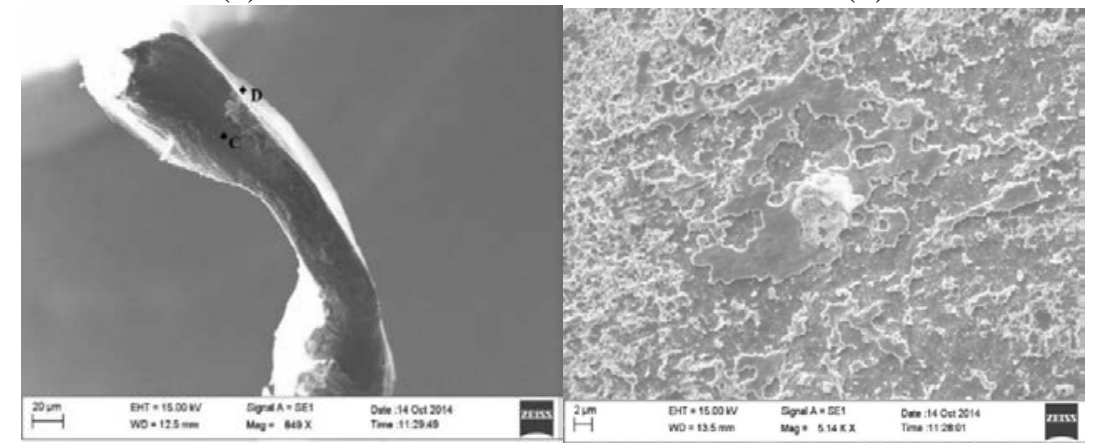

(c)

(d)

Fig 3 Thesurface morphology of the SEM for Failure 11\#. (a)(b)SEM image of the pressed mark of the second bond. (c) SEM image of bond wire for 11\#.(d)SEM amplificatory image of the pressed mark for 11\#.

The Fig 3(a) illustrated that the first bond was very good. The shallow pressed marks were seen from Figs 3(b) and 4(b). The Fig 3(c) and Fig 4(a)exhibited the bend wires off the pads.As Figs 3(d) and $4(\mathrm{c})$ were shown, there were many ring cavities around the bonding area at the pads where the second bond were bonded.

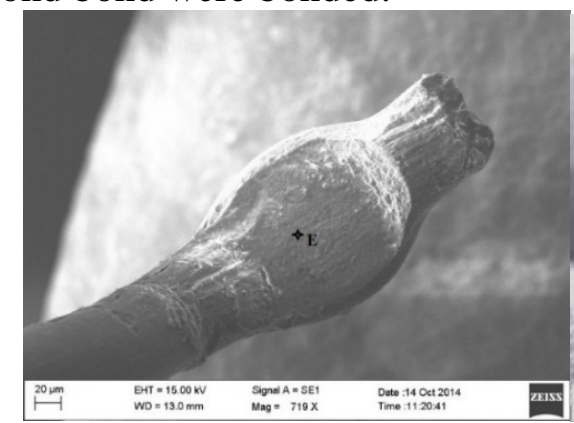

(a)

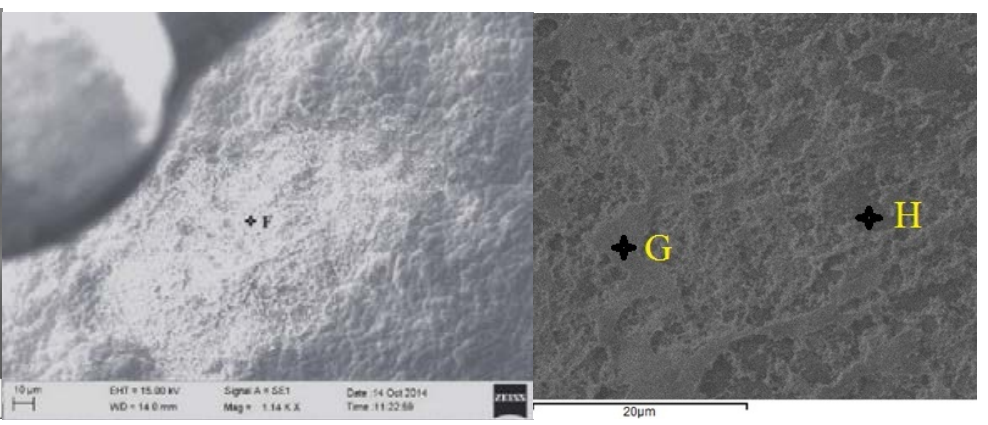

(b)

(c)

Fig 4 Thesurface morphology of the SEM for Failure8\#. (a) SEM image of a wedge bond for 8\#.(b)SEM image of the pressed mark of the second bond. (c) SEM image of bond wire for 8\#.

EDSAnalysis. For the special region, where was marked letters inFigs 3 and 4, EDS analysis were done. The results wereexhibited intable II.It can be concluded that the element composition of the second bond, the bonding region and the material of the bond wire for sample 11\# and 8\#.

Table 2 Theelement composition of the marker points

\begin{tabular}{cccccccc}
\hline \multirow{2}{*}{$\begin{array}{c}\text { Marker } \\
\text { points }\end{array}$} & \multirow{2}{*}{ Position } & \multicolumn{5}{c}{ The atomic percentage of each element (\%) } \\
& The bonding region of the pad (11\#) & 1.89 & 26.18 & - & 45.04 & 13.09 & 13.80 \\
$\mathrm{~A}$ & The pad except the bonding region (11\#) & - & 48.53 & - & 51.47 & - & - \\
$\mathrm{B}$ & The coating of the bond wire (11\#) & 87.52 & - & 1.35 & - & 11.14 & - \\
$\mathrm{C}$ & The bonding regionof the wire (11\#) & 9.29 & 16.78 & - & 38.20 & 30.67 & 5.06 \\
$\mathrm{D}$ & The coating of the bond wire (8\#) & 73.95 & - & 1.02 & 16.47 & 8.56 & - \\
$\mathrm{E}$ & The bonding region of the pad (8\#) & 2.43 & 26.52 & - & 48.18 & - & 22.87 \\
$\mathrm{~F}$ & The light bonding region of the pressed mark(8\#) & - & 45.26 & - & 46.05 & - & 8.70 \\
$\mathrm{G}$ & The darkbonding regionof the pressed mark(8\#) & 4.27 & 13.28 & - & 36.80 & - & 45.64 \\
$\mathrm{H}$ & & & & & & &
\end{tabular}


We can know that the components of bonding area A and F point of the pad were gold and some aluminum, nickel and carbon, but the sample 11\# had a little element of oxygen. And carbon accounted for nearly half of all elements. While the B point (the coating of the pad) was coated by gold and some carbon.That is to say, the material of the pad was gold. The table II also illustrated aluminum and a small number of silicon and were the components of the bond wire coating $\mathrm{C}$ and $\mathrm{E}$ points. The material of the wire was Si-Al. But the wire of sample 11\# also included carbon. However, the composition of $\mathrm{D}$ points in the bonding region of the bond wire was gold and a little nickel, aluminum, carbon and oxygen. As The table II shown, the components about $\mathrm{H}$ point of the dark position were a little bit different from $G$ point of the light position. There was a little aluminum besides a small amount of nickel besides and carbon of $\mathrm{H}$ point. The table II depicted that the pressed mark's components were more nickel and some carbon.

Analysis and Summary.By observing internal visual inspection, SEMexamination combined with EDS analysis, the summary can be drawn:

a) The type of the second bond point was wedge shape. The material of the wire was aluminum combined with little silicon, and the material of the pad was gold.

b) There was a little Nickel attached to the bonding area. Nickel may be an additive in in the process of wire bonding.

c) From the content of aluminum and gold elements in the bonding region, there was some change between gold and aluminum. Because of the different atomic diffusion coefficient between them, and the phenomenon that the mutual diffusion between gold and aluminum in high temperature was easily exist, especially after storage. The formation of the ring cavity around the wire bonding points called "Kirkendall" effect. At high temperatures, the diffusion of the gold is faster than the aluminum, which leads to the formation of intermetallic compounds ( $\mathrm{AuAl}, \mathrm{AuAl}_{2}$, $\mathrm{Au}_{2} \mathrm{~A} 1, \mathrm{Au}_{4} \mathrm{~A} 1, \mathrm{Au}_{5} \mathrm{Al}$ ) or form a gap in the gold aluminum interface to produce a hole. By the SEM images and bonding materials, we can know that the ring cavitieswereKirkendall holes. In this paper, the samples experienced the test at $125^{\circ} \mathrm{C}$ for 1000 hours. So the main reason to cause the Kirkendall holes was mostly the high temperature test.

d) From the EDS analysis,it was easily to see that there was a small proportion of carbon, oxygen, and nickel in the bond area, especially carbon. It was not accident to include the carbon and oxygen. Presumably during the bonding process, the bonding interface wasn't clean with alcohol, oil, dust and so on. The stain lead to the bond strength weak, and then influenced the reliability of the MOSFETs.

\section{Conclusions}

For semiconductor devices such as MOSFETs, gold-aluminum bonding was often used. The right bonding strength forms a qualified bond point.

1) The composition of the bonding surface has some effect on the bonding strength. Don't use bonding material like gold aluminum which does easily to produce intermetallic compound material, unless necessary.

2) And it is necessary to clean the bond pads and surface of the bonding wire after the process and before the package. The carbon oxygen compoundis enemy for aluminum, which is the easily oxidized material.

3) The high temperature is a key factor to affect the bonding strength. Of course, it is important to strictly control the temperature, whatever condition it is.

\section{Acknowledgment}

This work was supported by technical basis research projects in Science and Industry Bureau of China under Grant No.Z132012A001 and Z1320130013, and Natural Science Foundation of China under Grant No.61201028 and 61376042. 


\section{References}

[1] Huangkuang Kung,Bowun Huang and Hsiangchen Hsu, The effect of cross-section geometry of bond wire on wire sweep for semiconductor packages5th International Microsystems Packaging Assembly and Circuits Technology Conference (IMPACT). Taipei(2010)1-4.

[2] Qin, I.,Hui Xu, Milton, B.,Clauberg, H.,Chylak, B., Abe, H.,Dongchul Kang, Endo, Y., Osaka and M., Nakamura, S., Molded reliability study for different $\mathrm{Cu}$ wire bonding configurations,63rd Electronic Components and Technology Conference (ECTC). Las Vegas. (2013) 1587-1594.

[3] Yuqing Chao, Zhaojian Yang andHailingQiao, Progress on Technology of Wire Bonding,Electronics Process Technology.04 (2007) 205-210.(in Chinese)

[4] Cheng Gao, ShenglongDiao and Jiaoying Huang,Failure mechanism analysis of multilayer chip inductors,Journal of Convergence Information Technolog. 7,18 (2012)574-583.

[5] Jiaoying Huang, Cheng Gao, Jinyan Chen and Yue Sun, Mechanism analysis of aluminum corrosion and its application to switch transistor,International Journal of Advancements in Computing Technology. 4,20 (2012) 222-229.

[6] WeilianSun ,ZailiangChen and ChenbiaoWang, Mechanical failure analysis and failure case studies Ideas, Heat Treatment of Metals.01 (2004) 69-73,86.

[7] Quan Wang, Jianning Ding and Wenxiang Wang, A Novel Instrument of Thermo Compression Wire Bonding, The seventh Academic Symposium of China micro and neon technology, China. (2005)3.

[8] YianliangKuo, Lin Y.Cand Lin Y.-T, Failure analysis of IMCcracking and voiding induced by molding compound voids in advanced wire bonding packages,Proceedings of the 11th International Symposium on the Physical and Failure Analysis of Integrated Circuits, Taiwan.(2004) 221, 224.

[9] Yeohlai Seng, Characterization of Intermetallic Growth for Gold Bonding and Copper Bonding on Aluminum Metallization in Power Transistors,9th Electronics Packaging Technology Conference, Singapore. (2007) 731, 736.

[10]R. Klengel, S.Bennemann and J.Schischka, Advanced failure analysis methods and microstructural investigations of wire bond contacts for current microelectronic system integration,European Microelectronics and Packaging Conference,European.(2009) 1, 6.

[11]HyoungJoon Kim, Min-Seok Song, Kyung-Wook Paik,Jeong-TakMoon and Jun-Yeob Song, Investigation of interfacial phenomena of alloyed Au wire bonding, 15th Electronics Packaging Technology Conference (EPTC 2013), Chengdu. (2013) 479-482.

[12]YU Min, Discussion on the Characteristics of the Switch of the Field Effect Transistors, Chinese New Technologies and Products.02(2015)32.(in Chinese) 